\title{
Thermodynamic properties of a magnetically modulated graphene
}

\author{
SK Firoz Islam, Naveen K. Singh and Tarun Kanti Ghosh \\ Department of Physics. Indian Institute of Technology-Kanpur, Kanpur-208 016, India
}

(Dated: November 19, 2018)

\begin{abstract}
The effect of magnetic modulation on thermodynamic properties of a graphene monolayer in presence of a constant perpendicular magnetic field is reported here. One-dimensional spatial electric or magnetic modulation lifts the degeneracy of the Landau levels and converts into bands and their bandwidth oscillates with magnetic field leading to Weiss-type oscillation in the thermodynamic properties. The effect of magnetic modulation on thermodynamic properties of a graphene sheet is studied and then compared with electrically modulated graphene and magnetically modulated conventional two-dimensional electron gas (2DEG). We observe Weiss-type and de Haas-van Alphen (dHvA) oscillations at low and high magnetic field, respectively. There is a definite phase difference in Weiss-type oscillations in thermodynamic quantities of magnetically modulated graphene compared to electrically modulated graphene. On the other hand, the phase remains same and amplitude of the oscillation is large when compared with the magnetically modulated 2DEG. Explicit asymptotic expressions of density of states and the Helmholtz free energy are provided to understand the phase and amplitude of the Weiss-type oscillations qualitatively. We also study thermodynamic properties when both electric and magnetic modulations are present. The Weiss-type oscillations still exist when the modulations are out-of-phase.

PACS numbers: $65.80 .+\mathrm{n}, 71.70 . \mathrm{Di}, 71.18 .+\mathrm{y}$
\end{abstract}

\section{INTRODUCTION}

Graphene is a two-dimensional sheet of carbon allotrope with honeycomb lattice structure. It can be considered as a basic building block of all other dimensional carbon allotrope $\mathrm{e}^{-4}$. The relativistic-like, massless and linear energy spectrum of graphene's quasi-particles in low-energy range close to the Dirac points in it's band structure reflect in it's different properties like transport properties, optical properties, magnetic properties etc in different way than the conventional 2DEG formed in the semiconductor heterostructures. The massless linear energy dispersion and charge conjugation symmetry cause some unusual phenomena like Klein paradox, anomalous quantum Hall effect and non-zero Berry phase ${ }^{-} \underline{-9}$.

Effect of electric or/and magnetic modulations on transport and thermodynamic properties of quantum 2DEG systems is continuing to be an active research field. The magnetotransport properties of a conventional 2DEG in presence of a one-dimensional (1D) weak electric modulation have been studied in great details experimentally and theoretically by various groups ${ }^{10}-15$. Later, the magnetotransport properties of a magnetically modulated 2DEG in presence of a constant perpendicular magnetic field have been studied theoretically $\underline{16}-19$ and also experimentally ${ }^{20}-22$. The presence of weak electric/magnetic modulation broadens the Landau energy levels into bands. The band width oscillates with the magnetic field and its oscillations are also reflected in magnetotransport properties. It has been observed that the magnetoresistivity tensor oscillates with inverse of the magnetic field at very low magnetic field. This oscillation is known as the Weiss oscillation which is completely different from the Shubnikov-de Hass (SdH) oscillations observed at higher magnetic field. Period of
Weiss oscillation varies with electron density $n_{e}$ as $\sqrt{n}_{e}$, whereas for SdH it depends linearly on $n_{e}$. The Weiss oscillation is due to effect of the commensurability between two length scales in the system: the cyclotron diameter at the Fermi energy and the period of the modulated electric/magnetic potential. Magnetotransport properties of electrically ${ }^{23,24}$ and magnetically modulated graphene ${ }^{25}$ in presence of a constant perpendicular magnetic field have been studied recently.

The effect of weak electric and magnetic modulation on thermodynamic properties for 2DEG in presence of a perpendicular magnetic field have been studied theoretically ${ }^{12,26,27}$. It is observed that the Weisstype oscillation in various thermodynamic properties are present. The Weiss-type oscillations are completely different in origin from the usual de Haas-van Alphen (dHvA) oscillations which occurs at high magnetic field. The dHvA oscillation is effect of the formation of discrete Landau energy levels due to the quantizing magnetic field. Recently, thermodynamic properties of a monolayer graphene in presence of weak electric modulation have been studied and the Weiss-type oscillations are seen 28 . These results inspired us to study thermodynamic properties of a magnetically modulated graphene sheet in presence of a constant magnetic field.

The source of the magnetic modulation, magnetic stripes or superconducting materials, acts like electrical gates and produces an electric modulation. The transport properties of a $2 \mathrm{DEG}^{16}$ and a graphene sheet ${ }^{29}$ in presence of both the modulations were studied.

In this paper we study the effect of magnetic modulation on thermodynamic properties of a graphene sheet in presence of a constant magnetic field. We compare our results with electrically modulated graphene and with magnetically modulated conventional 2DEG. We also calculate an asymptotic expression of density of states (DOS) 
and the Helmholtz free energy of a magnetically modulated graphene in presence of a constant magnetic field at low temperature. In addition to these, we also study thermodynamic properties of graphene and 2DEG when both electric and magnetic modulations are present.

This paper is organized as follows. In section II, we summarize the standard results of the energy spectrums and the corresponding eigenstates for electrically and magnetically modulated graphene layer and 2DEG in presence of a constant magnetic field. In section III, we numerically calculate the thermodynamic quantities like, Helmholtz free energy, internal energy, entropy, heat capacity and magnetization. We analyze our numerical results and compare with electrically modulated graphene and magnetically modulated 2DEG in section IV. We discuss the behavior of the asymptotic expression of the DOS and the Helmholtz free energy for magnetically modulated graphene in section V. In section VI, we study thermodynamic properties of graphene and 2DEG in presence of both electric and magnetic modulations. We present summary of our work in section VII. The detail calculation of the DOS by using Green's function method is presented in the Appendix 1.

\section{ENERGY SPECTRUM}

We consider a monolayer graphene sheet subjected to a perpendicular constant magnetic field $\mathbf{B}_{0}=B_{0} \hat{z}$, the Hamiltonian of an electron with charge $-e$ is given by

$$
H_{0}^{G}=v_{F} \boldsymbol{\sigma} \cdot\left(\mathbf{p}+e \mathbf{A}_{0}\right)
$$

where $\boldsymbol{\sigma}=\left(\sigma_{x}, \sigma_{y}\right)$ are the Pauli matrices, $v_{F} \approx 10^{6}$ $\mathrm{m} / \mathrm{s}$ is the Fermi velocity and $\mathbf{A}_{0}$ is the magnetic vector potential. Here, we have chosen the Landau gauge $\mathbf{A}_{0}=B_{0} x \hat{y}$. The energy eigenvalues are $E_{n}^{g}=\hbar \omega_{g} \sqrt{2 n}$, where $n=0,1,2,3 \ldots$ is the Landau level index and $\omega_{g}=v_{F} \sqrt{e B_{0} / \hbar}$ is the cyclotron frequency. The corresponding normalized eigenstates are

$$
\Psi_{n, k_{y}}(x, y)=\frac{e^{i k_{y} y}}{\sqrt{2 L_{y} l_{0}}}\left[\begin{array}{c}
-i \phi_{n-1}\left[\left(x+x_{0}\right) / l_{0}\right] \\
\phi_{n}\left[\left(x+x_{0}\right) / l_{0}\right]
\end{array}\right],
$$

where $\phi_{n}(x)$ is the known harmonic oscillator wave function, $l_{0}=\sqrt{\hbar /\left(e B_{0}\right)}$ is the magnetic length, $x_{0}=-k_{y} l_{0}^{2}$ is the center of the cyclotron orbit and $L_{y}$ is the width of the graphene in the $y$-direction.

We consider the perpendicular magnetic field is modulated very weakly by $\mathbf{B}_{\mathbf{1}}=B_{1} \cos (q x) \hat{z}$, where $q=2 \pi / a$ and $a$ is the modulation period. The total Hamiltonian can be written as $H=H_{0}^{G}+H_{1}$, where $H_{1}=$ $V_{m}^{g} \sigma_{y} \sin (q x)$. Here, with $V_{m}^{g}=e v_{F} B_{1} / q$ is the strength of the effective magnetic potential determined by the amplitude $B_{1}$ and the period $a$ of the magnetic modulation. Using the first-order perturbation theory, the energy correction to the unperturbed Hamiltonian $H_{0}^{G}$ is given as ${ }^{25}$

$$
\Delta E_{n, k_{y}}^{g, m}=V_{m}^{g} \sqrt{\frac{n}{u}} e^{-u / 2}\left[L_{n-1}(u)-L_{n}(u)\right] \cos \left(q x_{0}\right)(3)
$$

where $L_{n}(u)$ is the Laguerre polynomial and $u=q^{2} l_{0}^{2} / 2$. So the total energy upto the first-order in $V_{m}^{g}$ is given by $E_{n, k_{y}}^{g, m}=E_{n}^{g}+\Delta E_{n, k_{y}}^{g, m}$. The band width in presence of the magnetic modulation is $\Delta_{m} \sim \sin (2 \sqrt{n u}-\pi / 4)$. Using the flat band condition, we get $2 R_{c}=a(j+1 / 4)$, with $j=0,1,2,3, \ldots$ and $R_{c}=k_{F} l_{0}^{2}$.

The energy correction due to the weak electric modulation $U(x)=V_{e}^{g} \cos (q x)$ can be obtained by the same method and it is given as ${ }^{23}$

$$
\Delta E_{n, k_{y}}^{g, e}=\frac{V_{e}^{g}}{2} e^{-u / 2}\left[L_{n}(u)+L_{n-1}(u)\right] \cos \left(q x_{0}\right) .
$$

So the total energy upto the order of $V_{e}^{g}$ is $E_{n, k_{y}}^{g, e}=$ $E_{n}^{g}+\Delta E_{n, k_{y}}^{g, e}$. The bandwidth in presence of the electric modulation is $\Delta_{e} \sim \cos (2 \sqrt{n u}-\pi / 4)$. The bandwidths due to magnetic modulation and due to the electric modulation are out of phase. The band will be flat when $2 R_{c}=a(j+3 / 4)$ which is different from what we get in the magnetic modulation case.

The Hamiltonian of a conventional 2DEG in presence of a perpendicular constant magnetic field $\mathbf{B}_{\mathbf{0}}$ is

$$
H_{0}^{2 d}=\frac{p_{x}^{2}}{2 m^{*}}+\frac{1}{2 m^{*}}\left(p_{y}+e A_{y}\right)^{2} .
$$

The energy spectrum is $E_{n}^{2 d}=\hbar \omega_{c}(n+1 / 2)$, where $n=$ $0,1,2,3 \ldots$ and $\omega_{0}=e B_{0} / m^{*}$ is the cyclotron frequency. The corresponding eigenstates are

$$
\Psi_{n, k_{y}}(x, y)=\frac{e^{i k_{y} y}}{\sqrt{L_{y} l_{0}}} \phi_{n}\left[\left(x+x_{0}\right) / l_{0}\right] .
$$

In presence of the weak magnetic modulation $\mathbf{B}_{1}$, the total Hamiltonian is $H=H_{0}^{2 d}+H_{1}^{2 d}$, where $H_{1}^{2 d}=$ $\left[V_{m}^{2 d} /(\hbar q)\right]\left(p_{y}+e B_{0} x\right) \sin (q x)$ with $V_{m}^{2 d}=\hbar\left(e B_{1} / m^{*}\right)$ is the strength of the effective magnetic potential determined by the amplitude of the magnetic modulation. The first-order energy correction due to the weak magnetic modulation is given by $\underline{16,17}$

$$
\begin{aligned}
\Delta E_{n, k_{y}}^{2 d, m} & =V_{m}^{2 d} e^{-\frac{u}{2}}\left[\frac{u-2 n}{2 u} L_{n}(u)\right. \\
& \left.+\frac{n}{u} L_{n-1}(u)\right] \cos \left(q x_{0}\right) .
\end{aligned}
$$

The total energy of a 2DEG in presence of the magnetic modulation is then $E_{n, k_{y}}^{2 d, m}=E_{n}^{2 d}+\Delta E_{n, k_{y}}^{2 d, m}$. The energy correction due to electric modulation is given by

$$
\Delta E_{n, k_{y}}^{2 d, e}=\frac{V_{e}^{2 d}}{2} e^{-u / 2}\left[L_{n}(u)+L_{n+1}(u)\right] \cos \left(q x_{0}\right) .
$$

All these standard results will be used to calculate the thermodynamic properties numerically in the next section.

\section{THERMODYNAMIC QUANTITIES}

In this section we discuss all standard thermodynamic equations to be used for calculating chemical potential, 
Helmholtz free energy, internal energy, entropy, magnetization and specific heat.

The DOS of a magnetically modulated graphene sheet in presence of a constant magnetic field can be written as

$$
D(E)=\frac{A}{\pi l_{0}^{2}} \sum_{n, k_{y}} \delta\left(E-E_{n, k_{y}}\right),
$$

where $A=L_{x} L_{y}$ is the area of the graphene sheet and $E_{n, k_{y}}$ is a energy dispersion of a given system like $E_{n, k_{y}}^{g, m}, E_{n, k_{y}}^{g, e}, E_{n, k_{y}}^{2 d, m}$. The dependence of chemical potential $\mu(B, T)$ on temperature $(T)$ and magnetic field $(B)$ can be obtained numerically by using the following normalization condition

$$
N=\int_{0}^{\infty} D(E) f(E) d E
$$

where $N$ is the total number of electrons, $f(E)=$ $\left[\exp \left(\frac{E-\mu}{k_{B} T}\right)+1\right]^{-1}$ is the Fermi-Dirac distribution function and $k_{B}$ is the Boltzmann constant. Using the expression of $D(E)$ given in the above equation we get

$$
n_{e} \pi l_{0}^{2}=\frac{1}{\pi} \sum_{n=0}^{\infty} \int_{0}^{\pi} d t f\left(E_{n, t}\right),
$$

where $n_{e}$ is the electron density and $t=q x_{0}$.

The total internal energy can be written as

$$
U=\int_{0}^{\infty} E D(E) f(E) d E .
$$

The internal energy per unit area is

$$
\frac{U}{A}=\frac{1}{\pi^{2} l_{0}^{2}} \sum_{n=0}^{\infty} \int_{0}^{\pi} E_{n, t} f\left(E_{n, t}\right) d t
$$

Now for a system of non-interacting electrons, the Helmholtz free energy density ${ }^{30}$ is given by

$$
\frac{F}{A}=\mu n_{e}-\frac{k_{B} T}{\pi} \sum_{n=0}^{\infty} \int_{0}^{\pi} \ln \left[1+\exp \left(\frac{\mu-E_{n, t}}{k_{B} T}\right)\right] d t .
$$

From the above equations it is clear that the DOS plays an important role in the behavior of the thermodynamic properties. In presence of the perpendicular magnetic field the DOS shows a series of delta function because of the quantized energy spectrum. Graphene and conventional 2DEG having different energy spectrums reflects differently in their thermodynamic properties. By using the above results we compute entropy via $S=(U-F) / T$, orbital magnetization $M=-\left(\partial F / \partial B_{0}\right)_{A, N}$ and heat capacity $C=T(\partial S / \partial T)_{A, N}=-T\left(\partial^{2} F / \partial^{2} B_{0}\right)_{A, N}$. For better visualization of effect of the magnetic modulation we plot the fluctuation $\Delta \Pi=\Pi\left(B_{1}\right)-\Pi\left(B_{1}=0\right)$, where $B_{1}$ is the strength of magnetic modulation, and $\Pi$ is a thermodynamic quantity like $\mu, F, U, M, S, C$.

\section{NUMERICAL RESULTS AND DISCUSSIONS}

Thermodynamic properties of magnetically modulated graphene sheet in presence of a constant magnetic field are studied. The aim is to study the effect of magnetic modulation on graphene in comparison with the electrically modulated graphene and magnetically modulated conventional 2DEG. We plot the fluctuation due to weak modulation in chemical potential, Helmholtz free energy, magnetization, internal energy, entropy and specific heat with the magnetic field $B_{0}$. We have used the following parameters for numerical calculation: electron density $n_{e}=3.16 \times 10^{15} / \mathrm{m}^{2}$, effective mass of an electron $m^{*}=0.067 m_{e}$ with $m_{e}$ is the bare electron mass, temperature $T=2 \mathrm{~K}$, modulation period $a=382 \mathrm{~nm}$ as used in ${ }^{26,28}$. For these parameters, $E_{F}^{g}=0.1 \mathrm{eV}$ and $E_{F}^{2 d}=14.25 \mathrm{meV}$.

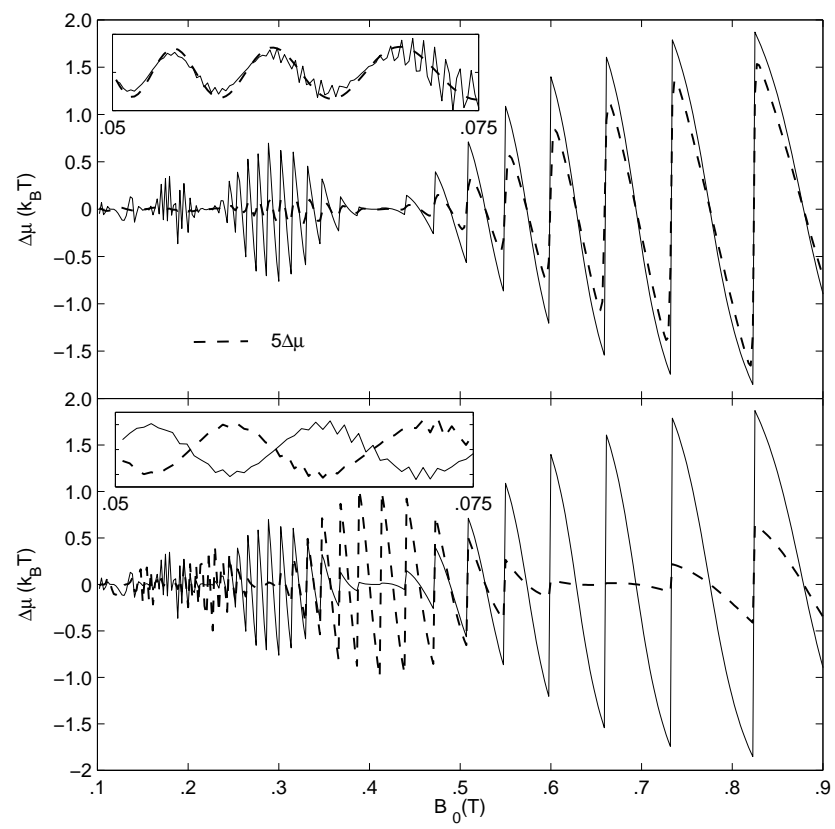

FIG. 1: Plots of the fluctuation in chemical potential vs magnetic field at $T=2 \mathrm{~K}$.

In figures 1-6, we have plotted the fluctuations in various thermodynamic properties, $\Delta \Pi$, due to both magnetic and electric modulations. We have scaled all the thermodynamic quantities per electron. Each figure contains two panels, the upper panel shows the effect of the magnetic modulation on graphene (solid line) and conventional 2DEG (dashed line), and the lower panel shows this fluctuation for the electrically modulated (dashed line) and magnetically modulated (solid line) graphene. We have zoomed the oscillations at low magnetic field and shown in the inset of figures 1-6. It clearly shows that weak $1 \mathrm{D}$ periodic potential, either electric or magnetic in nature, induces new oscillations at low magnetic 


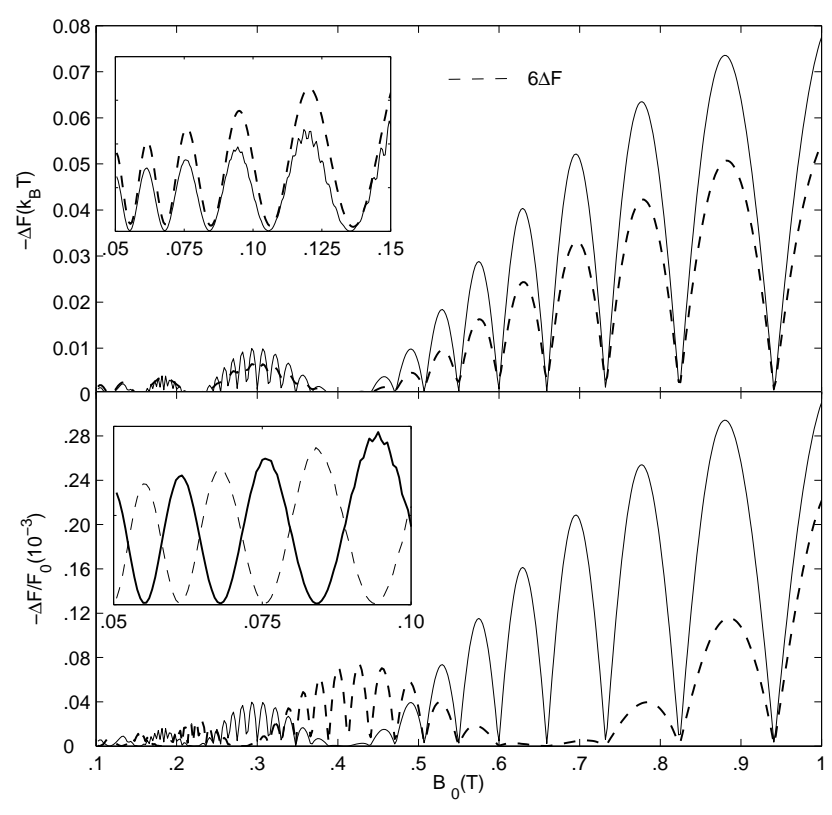

FIG. 2: Plots of the change in the free energy vs magnetic field. To make the fluctuation dimensionless we use $F_{0}=$ $E_{F}^{2 d} / 2$ in the lower panel but as $E_{F}$ is different for the two systems we are showing fluctuation in units of $k_{B} T$ in the upper panel.

field. These modulation induced oscillation is due to the commensurability of the two length scales present in the system. These oscillations are similar to the Weiss oscillations observed in the magnetoresistance at low magnetic fields.

It is clear from the upper panels of the figures 1-6 that the fluctuation in the thermodynamic properties, $\Delta \Pi$, of the graphene sheet is quite large compared to the 2DEG system. This can be understood qualitatively from the following arguments. The energy correction for the magnetically modulated graphene and 2DEG systems are $V_{m}^{g}=1 \mathrm{meV}$ and $V_{m}^{2 d}=0.046 \mathrm{meV}$ for the same strength of the magnetic modulation $B_{1}=0.02 \mathrm{~T}$. Clearly, the energy correction due to the magnetic modulation in graphene is quite large compared to that of the 2DEG. This is the origin for higher amplitude fluctuation of the Weiss-type and dHvA oscillations in graphene compared to the 2DEG. It is interesting to note that amplitude of diffusive conductivity in magnetically modulated graphene is small compared to magnetically modulated $2 \mathrm{DEG} 25$.

The lower panels of the figures 1-6 show that the Weisstype oscillation has a definite phase difference in the thermodynamic properties between magnetic and electric modulation cases, which is due to the following reasons. The phase difference in the fluctuations between electrically and magnetically modulated graphene comes from the nature of the energy correction. In the electric modulation case the energy correction contains addition of the two successive Laguerre polynomials and in magnetic modulation case it is subtraction of the two successive Laguerre polynomials, giving rise to the cosine and sine term, respectively. We also observe that the dHvA-type oscillations for the two different kind of modulations remain in the same phase with each other. The phase difference between the two different kind of modulated systems are shown explicitly by using the analytical expressions of the DOS in the next section.

Figures (1) and (3) shows the fluctuation in chemical potential and magnetization with magnetic field, respectively. The fluctuation in magnetically modulated graphene is several times higher than the magnetically modulated 2DEG in the Weiss-type oscillation, but their phases remain same. On the other hand, the magnetically modulated graphene shows $\pi$ phase difference in Weiss-type oscillation compared to the electrically modulated graphene with the same amplitude.

Figure (2) is showing the fluctuation in the Helmholtz free energy where lower panel shows $\pi / 2$ phase differences between electric and magnetic modulation cases in graphene with the same amplitude. The amplitude of fluctuation of graphene is several times higher than the 2DEG but the phases remain same. From the flatband condition, the minima of the bandwidth occur at $B(T)=0.092,0.113,0.148,0.214,0.386$. On the other hand, the fluctuation in the free energy vanishes at $B(T)=0.092,0.115,0.150,0.214,0.385$. It shows that the minima of the free energy fluctuation occur at those values of the magnetic field where the bandwidth minima occur.

We have plotted internal energy fluctuation in Fig. (4). The lower panel of Fig. (4) shows that the Weiss-type oscillation is appeared with same amplitude but $\pi / 2$ phase difference when compared with the electrical modulation case. When we compare with conventional 2DEG, it is similar to the case of the Helmholtz free energy i. e. amplitude is higher in magnitude.

Figures (5) and (6) are showing the entropy and specific heat fluctuation, respectively. The fluctuation in graphene is higher by several times than that of the 2DEG. The phase relationship is not discernible in these figures.

In all the above cases, dHvA-type oscillation remains in the same phase and does not depend on the modulation type as it is the manifestation of the quantized Landau levels rather than periodic perturbation. Figure (7) shows the damping of fluctuation in chemical potential and free energy with increasing temperature. Temperature dependence in Weiss-type and dHvA oscillations are independent of type of modulation and already discussed in electrical modulation case. The phase relationships for all the thermodynamic quantities are given in Table (1).

Even at higher magnetic field, the effect of modulation on the fluctuation of thermodynamic quantities is still 


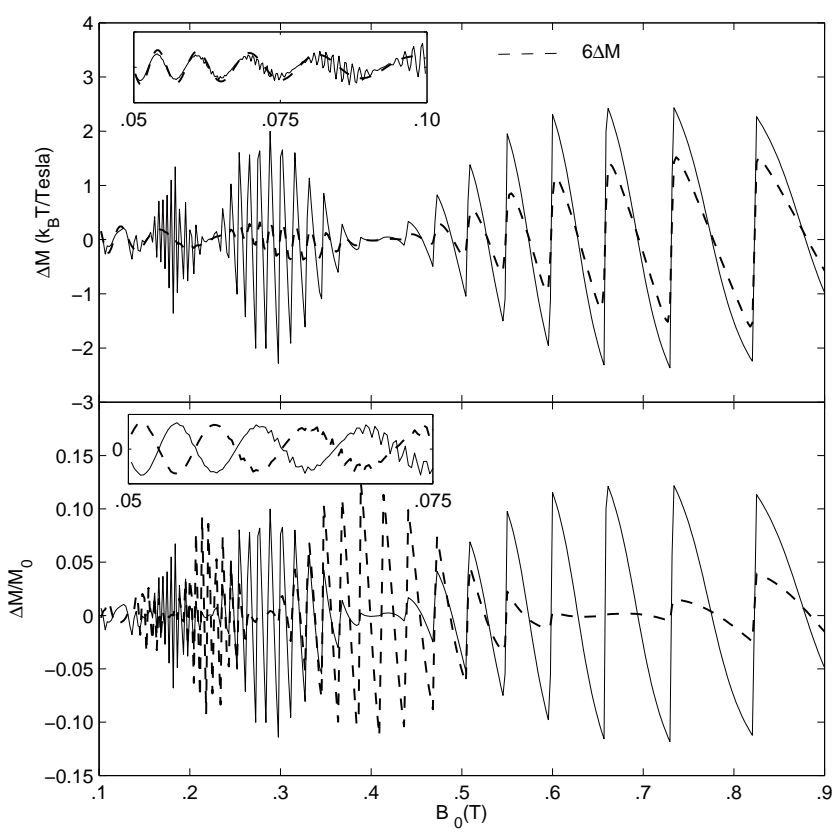

FIG. 3: Plots of the fluctuation in magnetization vs magnetic field. In the lower panel $\Delta M$ is scaled by $M_{0}=\mu_{B}^{*}$, where $\mu_{B}^{*}=e \hbar / 2 m_{g}$ is the effective Bohr magneton with $m_{g}=$ $E_{F}^{g} / v_{F}^{2}$ is the cyclotron mass.

\begin{tabular}{|c|c|c|c|c|c|c|}
\hline \hline Case & $\Delta \mu$ & $\Delta F$ & $\Delta M$ & $\Delta U$ & $\Delta S$ & $\Delta C$ \\
\hline Phase shift & $\pi$ & $\pi / 2$ & $\pi$ & $\pi / 2$ & indiscernible & indiscernible \\
\hline
\end{tabular}

TABLE I: The phase shifts in the Weiss-type oscillations appear in the fluctuation of thermodynamic quantities between the electrically and magnetically modulated graphene.

exist which is the manifestation of the modulated density of states. The dHvA-type oscillation is corresponding to the crossing of each Landau level one by one through the Fermi level. The density of available states per Landau level is $1 / 2 \pi l_{0}^{2}$ and it increases linearly with magnetic field which results in increasing amplitude of $\mathrm{dHvA}$ oscillation with magnetic field.

\section{ASYMPTOTIC RESULTS}

Here we derive an asymptotic expression for the DOS and the Helmholtz free energy. For weak magnetic modulation and under quasi-classical limit, we calculate the DOS by using the Green's function technique (see the Appendix 1) and written as a sum of the modulated and unmodulated part as $D(\epsilon)=D_{u}(\epsilon)+D_{m}(\epsilon)$, where

$$
D_{u}(\epsilon)=\frac{1}{\pi l_{0}^{2}} \frac{\epsilon}{\hbar \omega_{g}}\left[1+2 \cos \left(\pi \epsilon^{2}\right)\right]
$$

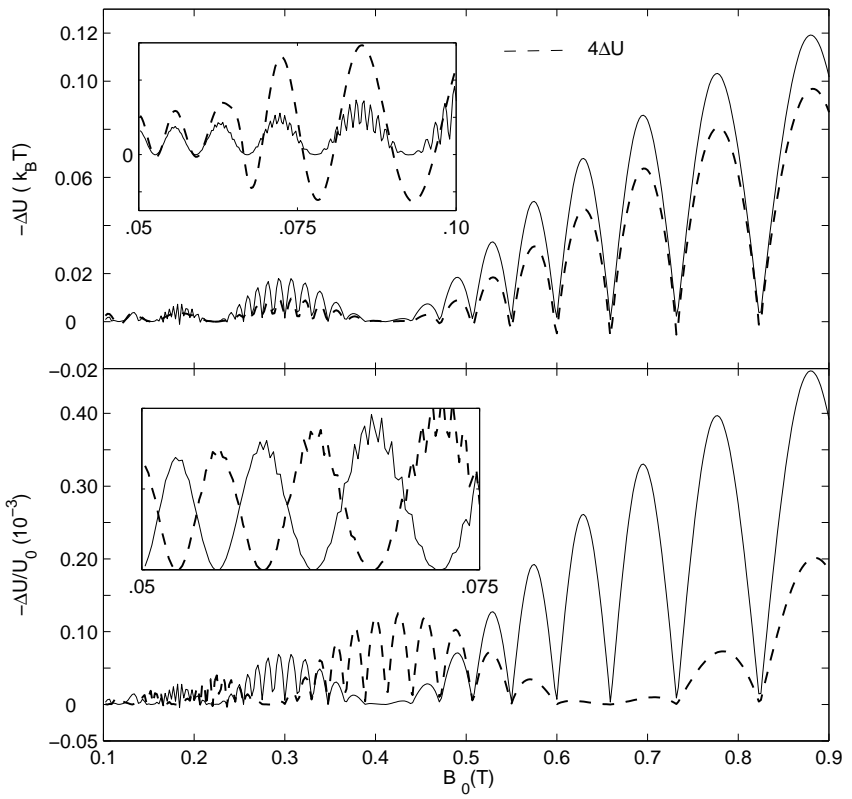

FIG. 4: Plots of the fluctuation in the internal energy vs magnetic field. In the lower panel $\Delta U$ is scaled by $U_{0}=E_{F}^{g} / 2$.

$$
D_{m}(\epsilon)=-\frac{2 \Omega_{m}}{\pi l_{0}^{2}} \frac{\epsilon}{\hbar \omega_{g}}\left[\epsilon^{3} \cos \left(\pi \epsilon^{2}\right) \sin ^{2}\left(q l_{0} \epsilon-\frac{\pi}{4}\right)\right],
$$

and

$$
\Omega_{m}=\frac{\left(V_{m}^{g}\right)^{2}}{\pi^{2}}\left(\frac{a}{l_{0}}\right)^{3}\left(\frac{1}{\hbar \omega_{g}}\right)^{3} \sin ^{2}\left(\frac{q l_{0}}{2 \epsilon_{F}^{g}}\right) .
$$

Here, $\epsilon=E /\left(\hbar \omega_{g}\right)$ and $\epsilon_{F}^{g}=E_{F}^{g} /\left(\hbar \omega_{g}\right)$.

The fluctuation in the DOS for electrically modulated graphene ${ }^{28}$ is proportional to $\cos ^{2}\left(q l_{0} \epsilon_{F}^{g}-\pi / 4\right)$. The appearance of the square of the sine term in Eq. (16) instead of square of cosine is the reason of definite phase differences in the fluctuation of all the thermodynamic quantities.

Using the two Eqs. (15) and (16) separately, we get an approximate analytical expression of the free energy and it's fluctuation. Our aim is to study the magnetic modulation effect in compare to the electrical modulation in graphene ${ }^{28}$. The change in the free energy due to the magnetic modulation can be expressed as

$$
\begin{aligned}
\frac{F_{m}}{A} & =-\left(k_{B} T\right) \hbar \omega_{g} \int_{0}^{\infty} D_{m}(\epsilon) \ln \left[1+\exp \left(\frac{\mu-\hbar \omega_{g} \epsilon}{k_{B} T}\right)\right] d \epsilon \\
& =-\Omega_{m} \frac{1}{\pi l_{0}^{2}} 4\left(\epsilon_{F}^{g}\right)^{4}\left(k_{B} T\right) \hbar \omega_{g} \sin ^{2}\left(q l_{0} \epsilon_{F}^{g}-\frac{\pi}{4}\right) \\
& \times \int_{0}^{\infty} \cos \left(\pi \epsilon^{2}\right) \ln \left[1+\exp \left(\frac{\mu-\hbar \omega_{g} \epsilon}{k_{B} T}\right)\right] d \epsilon .
\end{aligned}
$$

Under the assumption of very low temperature the above 


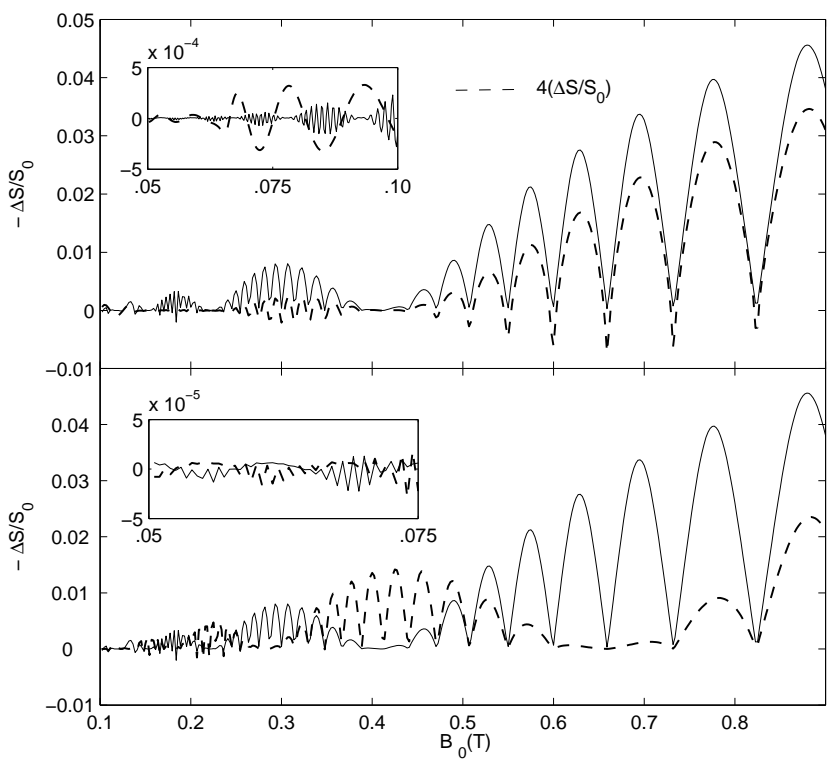

FIG. 5: Plots of the fluctuation in entropy vs magnetic field. In the lower panel $\Delta S$ is scaled by $S_{0}=k_{B}$.

integration results to

$$
\begin{aligned}
\frac{F_{m}}{A}= & -\frac{\Omega_{m}}{\pi l_{0}^{2}} \frac{\left(\hbar \omega_{g} \epsilon_{F}^{g}\right)^{4}}{\pi}\left\{\sin \left(\pi\left(\epsilon_{F}^{g}\right)^{2}\right)\right. \\
& \left.-\left(1-\frac{T / T_{g}^{d H v A}}{\sinh \left(T / T_{g}^{d H v A}\right)}\right) \frac{1}{2} \frac{\cos \left(\pi\left(\epsilon_{F}^{g}\right)^{2}\right)}{\left(\pi\left(\epsilon_{F}^{g}\right)^{2}\right)}\right\} \\
& \times \sin ^{2}\left(q l_{0} \epsilon_{F}^{g}-\frac{\pi}{4}\right)
\end{aligned}
$$

where $T_{g}^{d H v A}=\left(\hbar \omega_{g}\right) /\left(2 \pi^{2} k_{B} \epsilon_{F}^{g}\right)$ is the critical temperature for the dHvA-type oscillations in graphene. The ratio of amplitude of the free energy fluctuations of the magnetically and electrically modulated graphene is

$$
\frac{\lambda_{m}^{g}}{\lambda_{e}^{g}} \approx\left(\frac{V_{m}^{g}}{V_{e}^{g}}\right)^{2}
$$

Here, $\lambda_{m}^{g}$ and $\lambda_{e}^{g}$ are the amplitudes of free energy fluctuation for magnetically and electrically modulated graphene, respectively. The expression of $\lambda_{e}^{g}$ is taken from the Ref 30 . But in the case of conventional 2DEG this ratio has been calculated in Ref $\frac{26}{2}$ and it is given by

$$
\frac{\gamma_{m}^{2 d}}{\gamma_{e}^{2 d}}=\frac{1}{2 \pi^{2}}\left(\frac{E_{F}^{2 d}}{\epsilon_{a}}\right)\left(\frac{V_{m}^{2 d}}{V_{e}^{2 d}}\right)^{2}
$$

where $\epsilon_{a}=\hbar^{2} /\left(m^{*} a^{2}\right), \gamma_{m}^{2 d}$ and $\gamma_{e}^{2 d}$ are amplitudes of the free energy fluctuation for magnetic and electric modulation, respectively. The ratio of the amplitudes of different modulation cases in graphene behave differently from that of the 2DEG system.

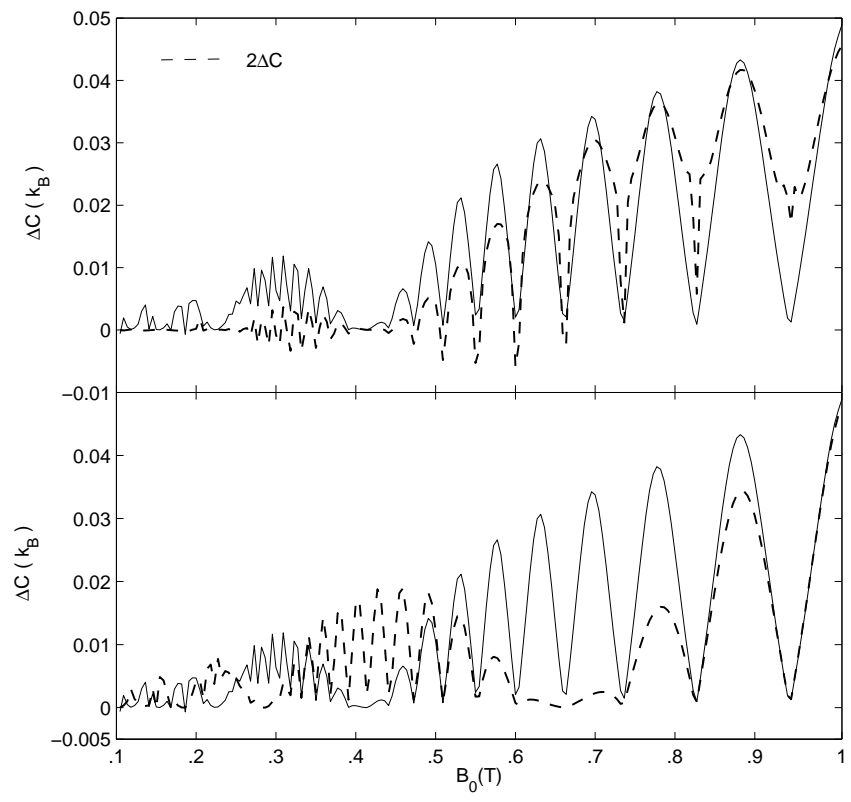

FIG. 6: Plots of the fluctuation in specific heat vs magnetic field.

Now we compare our result with the conventional 2DEG modulated magnetically. In this two case the ratio of amplitudes of the free energy fluctuations is given by

$$
\frac{\gamma_{m}^{2 d}}{\lambda_{m}^{g}}=\left(\frac{V_{m}^{2 d}}{V_{m}^{g}}\right)^{2} \frac{\pi}{\left(\beta E_{F}^{g}\right)^{2}}\left(\frac{a}{l_{0}}\right)^{2} \sqrt{\frac{E_{F}^{2 d}}{2 \hbar \omega_{0}}}\left(\frac{\omega_{g}}{\omega_{0}}\right)^{3}
$$

where $E_{F}^{g}$ is the Fermi energy of graphene whereas $E_{F}^{2 d}$ is for conventional 2DEG. Using $B_{0}=0.1 \mathrm{~T}, T=2 \mathrm{~K}$, $a=382 \mathrm{~nm}, \beta E_{F}^{g}=521, \omega_{g} / \omega_{0}=35$, we get

$$
\frac{\gamma_{m}^{2 d}}{\lambda_{m}^{g}} \sim 10^{2}\left(\frac{V_{m}^{2 d}}{V_{m}^{g}}\right)^{2}
$$

This equation leads to $\lambda_{m}^{g} \sim 4.7 \lambda^{2 d}$ when $B_{1}=0.02 \mathrm{~T}$. In graphene, the amplitude of free energy fluctuation in the Weiss-type oscillation is higher and in the same phase in compare to the conventional 2DEG.

\section{ELECTRIC AND MAGNETIC MODULATIONS}

In this section, we study how the thermodynamic properties discussed in the previous sections are changed in presence of an additional electric modulation with the same period. We consider two different cases: when both the modulations are in-phase and that are $\pi / 2$ outof-phase. The fluctuation in thermodynamic quantities like chemical potential and the Helmholtz free energy are 


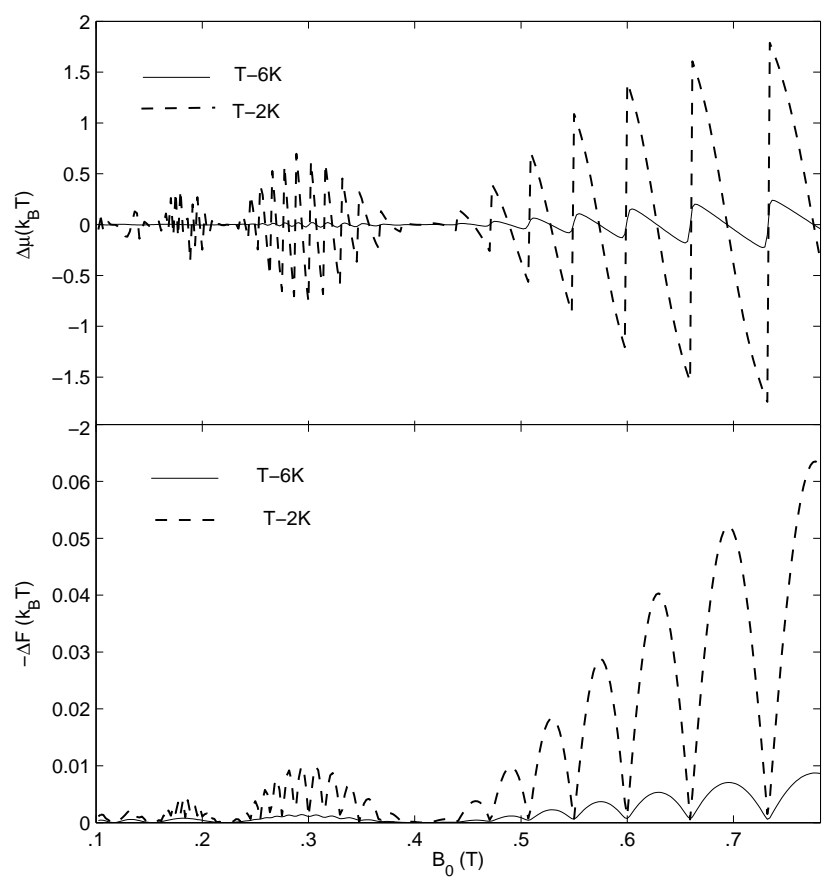

FIG. 7: Plots of the fluctuation in chemical potential and free energy vs magnetic field for $T=2 \mathrm{~K}$ and $T=6 \mathrm{~K}$ with the same parameter.

calculated numerically. Other thermodynamic quantities can easily be obtained by taking a suitable numerical derivative of the Helmholtz free energy fluctuation.

In-phase modulations: We consider a weak electric modulation described by the periodic potential $U(x)=$ $V_{e}^{g} \cos (q x)$, which is in-phase with the magnetic modulation $\mathbf{B}_{1}(x)=B_{1} \cos (q x) \hat{z}$. When both the modulations are in the same phase, the total energy correction for graphene in a weak magnetic field can be written as

$$
\Delta E^{g}=\sqrt{f_{m}^{2}+f_{e}^{2}} \sin \left(2 \sqrt{n u}-\frac{\pi}{4}+\delta_{i}^{g}\right) \cos \left(q x_{0}\right),
$$

where $f_{m}=\left(2 V_{m}^{g} / \sqrt{\pi}\right)\left(n / u^{3}\right)^{1 / 4} \sin (\sqrt{u / 4 n}), f_{e}=$ $\left(V_{e}^{g} / \sqrt{\pi}\right)(1 / n u)^{1 / 4} \cos (\sqrt{u / 4 n})$ and $\delta_{i}^{g}=\tan ^{-1}\left(f_{m} / f_{e}\right)$. The flat-band condition at the Fermi energy gives the positions of the minima in the free energy fluctuation as $B_{j}=2 p_{F} B_{a} /\left(j+\frac{1}{4}-\frac{\delta_{i}^{g}}{\pi}\right)$. Here, $j$ is an integer, $p_{F}=a k_{F}$ is a dimensionless momentum and $B_{a}=\hbar /\left(e a^{2}\right)$ is the characteristic magnetic field. In this case, $\delta_{i}^{g}=\pi / 4$ and then $B_{j}=2 p_{F} B_{a} / j$.

Similarly for conventional 2DEG, the total energy correction in the low magnetic field can be written as

$$
\Delta E^{2 d} \simeq \sqrt{w_{m}^{2}+w_{e}^{2}} \sin \left(2 \sqrt{n u}-\frac{\pi}{4}+\delta_{i}^{2 d}\right) \cos \left(q x_{0}\right),
$$

where $\left.w_{m} \simeq V_{m}^{2 d} \sqrt{n /(\pi u \sqrt{n u}}\right), w_{e} \simeq V_{e}^{2 d} /(\sqrt{\pi \sqrt{n u}})$ and $\delta_{i}^{2 d}=\tan ^{-1}\left(w_{m} / w_{e}\right)$. The flat-band condition at the Fermi energy is now $B_{j}=2 p_{F} B_{a} /(j+1 / 4-$ $\left.\tan ^{-1}\left\{V_{m}^{2 d} p_{F} /\left(2 \pi V_{e}^{2 d}\right)\right\}\right)$.

In figures 8 and 9 , we plot the chemical potential and free energy fluctuations in presence of both electric and magnetic modulations, respectively. The upper panel shows the thermodynamic fluctuations of graphene (solid) and 2DEG (dashed) when the modulations are inphase and the lower panel shows the thermodynamic fluctuations of graphene (solid) and 2DEG (dashed) when the modulations are out-of-phase.

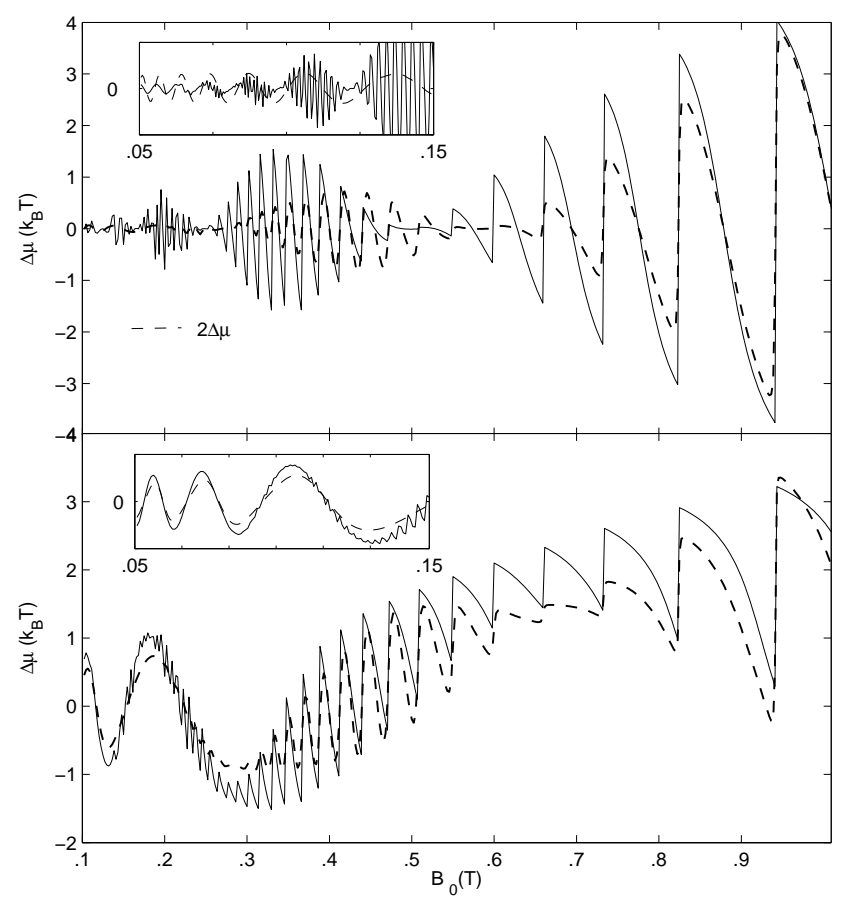

FIG. 8: Plots of the fluctuation in chemical potential vs magnetic field.

Out-of-phase modulation: We consider the same electric modulation $U(x)=V_{e} \cos (q x)$ and assume magnetic modulation is given by $\mathbf{B}_{1}(x)=B_{1} \sin (q x) \hat{z}$ so that the two modulations are $\pi / 2$ out-of-phase. To first-order in $V_{e}$ and $B_{1}$, the total energy correction for graphene can be written as

$$
\begin{aligned}
\Delta E^{g} & =f_{m} \sqrt{1+\left\{\left(\frac{f_{e}}{f_{m}}\right)^{2}-1\right\} \cos ^{2}(2 \sqrt{n u}-1)} \\
& \times \sin \left(q x_{0}+\delta_{o}^{g}\right),
\end{aligned}
$$

where $\tan \left(\delta_{o}^{g}\right)=\left(f_{m} / f_{e}\right) \tan (2 \sqrt{n u}-\pi / 4)$. Similarly, the total energy correction for $2 \mathrm{DEG}$ is written as

$$
\begin{aligned}
\Delta E^{2 d} & =w_{m} \sqrt{1+\left\{\left(\frac{w_{e}}{w_{m}}\right)^{2}-1\right\} \cos ^{2}(2 \sqrt{n u}-1)} \\
& \times \sin \left(q x_{0}+\delta_{o}^{2 d}\right)
\end{aligned}
$$

where $\tan \left(\delta_{o}^{2 d}\right)=\left(w_{m} / w_{e}\right) \tan (2 \sqrt{n u}-\pi / 4)$. In the case of graphene, though the band width is almost 
constant at the Fermi energy, but the magnetic field dependent phase factor $\delta_{o}^{g}$ plays an important role in the fluctuation of the thermodynamic quantities. This phase factor produces the Weiss-type oscillations in the thermodynamic properties at low magnetic field. In conventional 2DEG, the band width oscillates with the magnetic field when $w_{e} \neq w_{m}$ in our case but the Weisstype oscillation is due to the magnetic field dependent phase factor $\delta_{o}^{2 d}$ in the total energy correction. When $w_{e}=w_{m}$, the band width becomes non-oscillatory, but the Weiss-type oscillation still exist due to the magnetic field dependent phase factor. It is interesting to contrast our result with the results of the Weiss oscillations in the conductivity in presence of both the modulations 16.29 . The Weiss oscillation in conductivity is suppressed when the modulations are out-of-phase. On the other hand, the Weiss-type oscillations in the thermodynamic properties enhanced when the modulations are out-of-phase. In out-of-phase case, the Weiss-type oscillation in the thermodynamic quantities is due to the magnetic field dependent phase factor in the energy correction.

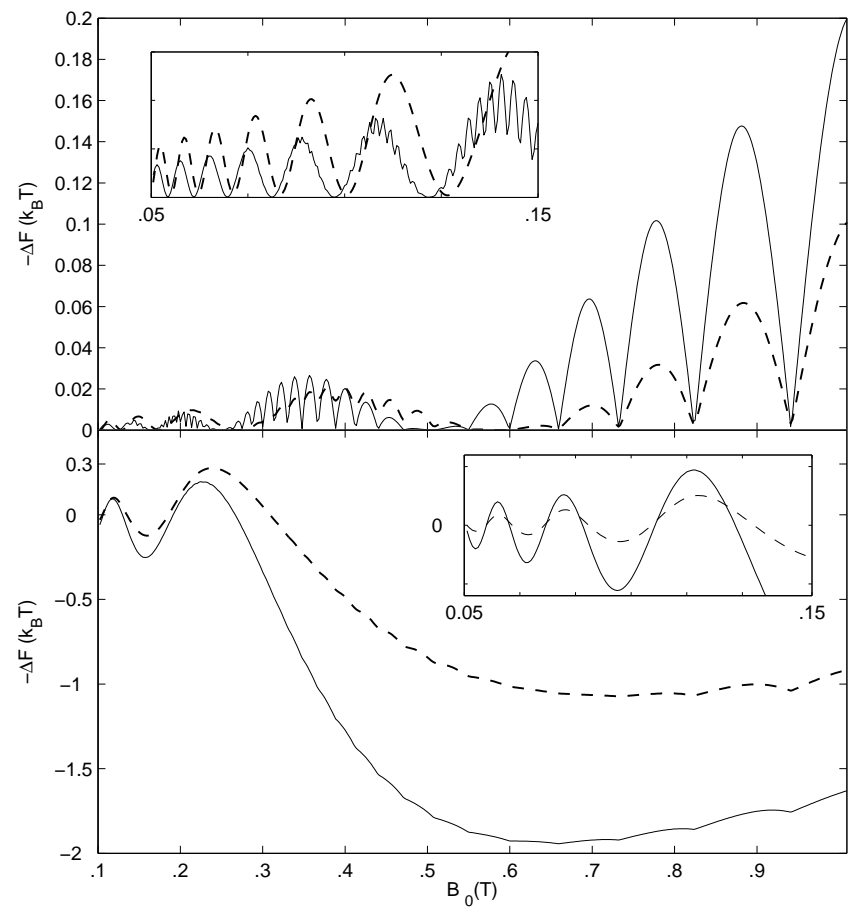

FIG. 9: Plots of the fluctuation in the Helmholtz free energy vs magnetic field.

\section{SUMMARY}

We have studied the effect of magnetic modulation on thermodynamic properties of a graphene sheet. The re- sults of magnetically modulated graphene are compared with electrically modulated graphene and magnetically modulated conventional 2DEG. It is observed that in the case of magnetically modulated graphene, a definite phase difference is appeared in the Weiss-type oscillation in compare to the electrically modulated graphene for all thermodynamic quantities. But when we compare our results with magnetically modulated conventional 2DEG, the amplitude of the fluctuations is found to be higher in graphene than 2DEG, but the phases remain same. We calculate the DOS and the Helmholtz free energy analytically. and explain the origin of the this phase difference in the Weiss-type oscillation. The enhancement of the fluctuation in magnetically modulated graphene in compare to the 2DEG and the definite phase difference between magnetically and electrically modulated graphene are explained by using the asymptotic results of the DOS in general and the Helmholtz free energy in particular.

We have also studied the thermodynamic properties like chemical potential and the Helmholtz free energy of graphene and conventional 2DEG in presence of both magnetic and electric modulations with the same period. The combined effect of both modulation does not modify the nature of the Weiss-type oscillation when they are in the same phase except a finite phase shift in the fluctuation. The effect of the out-of-phase modulations on thermodynamic fluctuations is different than that of inphase modulation. We found large amplitude Weiss-type oscillation at very low magnetic field even when bandwidth becomes non-oscillatory. For conventional 2DEG, effect of the out-of-phase modulation remains same as graphene though the bandwidth of 2DEG is oscillatory. This high amplitude Weiss-type oscillation is due to the magnetic field dependent phase factor which plays an important role here, unlike the Weiss oscillation in electrical transport properties where oscillation washed out for the same amplitude of energy correction due to two modulations.

\section{Acknowledgments}

This work is financially supported by C.S.I.R., Govt. of India under the grant CSIR-JRF-09/092(0687) 2009/EMR-1 F-O746.

\section{Appendix A}

Here we calculate the asymptotic expression of the DOS of graphene in presence of a modulated magnetic field: $\mathbf{B}=\left[B_{0}+B_{1} \cos (q x)\right] \hat{z}$. The total energy upto the first-order in $V_{m}^{g}$ is $E_{n, k_{y}}^{g, m}=\hbar \omega_{g} \sqrt{2 n}+G_{n} \cos \left(q x_{0}\right)$, where $G_{n}=V_{m}^{g} \sqrt{n / u} e^{-u / 2}\left[L_{n-1}(u)-L_{n}(u)\right]$ and $u=q^{2} l_{0}^{2} / 2$. Using $e^{-u / 2} L_{n}(u) \simeq(\pi \sqrt{n u})^{-1 / 2} \cos [2 \sqrt{n u}-\pi / 4]$ for higher values of $n$, we get the asymptotic expression of 
$G_{n}$ as

$$
\begin{aligned}
G_{n} & =\frac{2 V_{m}^{g}}{\sqrt{\pi}}\left(\frac{n}{u^{3}}\right)^{1 / 4} \sin \left(\sqrt{\frac{u}{4 n}}\right) \sin \left\{2 \sqrt{n u}-\frac{\pi}{4}\right\} \\
& =\frac{2 V_{m}^{g}}{\sqrt{\pi}}\left[\left(\frac{2 E}{q l_{0} \hbar \omega_{g}}\right)^{1 / 2} \frac{1}{q l_{0}}\right] \\
& \times \sin \left(\frac{q l_{0} \hbar \omega_{g}}{2 E}\right) \sin \left\{q l_{0} \frac{E}{\hbar \omega_{g}}-\frac{\pi}{4}\right\} .
\end{aligned}
$$

Now we use impurity broadened Landau levels in limiting case. The self-energy can be written as ${ }^{32}$

$$
\Sigma^{-}(E)=\Gamma_{0}^{2} \sum_{n} \int_{0}^{a} \frac{d x_{0}}{a} \frac{1}{E-E_{n, k_{y}}^{g, m}-\Sigma^{-}(E)}
$$

where $\Gamma_{0}$ is the broadening of the Landau levels due to impurities. By determining the imaginary part of the self-energy we can get the DOS through

$$
D(E)=\operatorname{Im}\left[\frac{\Sigma^{-}(E)}{\pi^{2} l_{0}^{2} \Gamma_{0}^{2}}\right] .
$$

By using residue theorem we get

$$
\begin{aligned}
\Sigma^{-}(E) & =2 \pi \Gamma_{0}^{2} \int_{0}^{a} \frac{d x_{0}}{a} \frac{E-\Sigma^{-}(E)-G_{n} \cos \left(q x_{0}\right)}{\left(\sqrt{2} \hbar \omega_{g}\right)^{2}} \\
& \times \cot \left[\frac{\pi}{\left(\sqrt{2} \hbar \omega_{g}\right)^{2}}\left\{E-\Sigma^{-}(E)-G_{n} \cos \left(q x_{0}\right)\right\}^{2}\right] \\
& \simeq \frac{\pi \Gamma_{0}^{2} E}{\left(\hbar \omega_{g}\right)^{2}} \int_{0}^{a} \frac{d x_{0}}{a} \times \\
& \times \cot \left[\frac{\pi E}{\left(\sqrt{2} \hbar \omega_{g}\right)^{2}}\left\{E-2 \Sigma^{-}(E)-2 G_{n} \cos \left(q x_{0}\right)\right\}\right] .
\end{aligned}
$$

Separating $\Sigma^{-}(E)$ into real and imaginary parts as

$$
\begin{aligned}
\Sigma^{-}(E) & =\Delta(E)+i \frac{\Gamma(E)}{2} \\
& =\frac{\pi \Gamma_{0}^{2} E}{\left(\hbar \omega_{g}\right)^{2}} \int_{0}^{a} \frac{d x_{0}}{a}\left[\frac{\sin u+i \sinh v}{\cosh v-\cos u}\right],
\end{aligned}
$$

where

$$
u=\frac{\pi E}{\left(\hbar \omega_{g}\right)^{2}}\left[E-2 \Delta(E)-2 G_{n} \cos \left(q x_{0}\right)\right]
$$

and $v=\frac{\pi E}{\left(\hbar \omega_{g}\right)^{2}} \Gamma(E)$. In the limit of large collisional broadening, $\pi \Gamma \gg \hbar \omega_{g}$, after expanding the hyperbolic term with respect to the small quantity $\exp (-v)$ up to first-order one obtains

$$
\begin{aligned}
\frac{\Gamma(E)}{2} & =\frac{\pi \Gamma_{0}^{2} E}{\left(\hbar \omega_{g}\right)^{2}}\left[1+2 \exp \left\{-\frac{\pi E \Gamma}{\left(\hbar \omega_{g}\right)^{2}}\right\}\right. \\
& \left.\times \int_{0}^{a} \frac{d x_{0}}{a} \cos \left\{\frac{\pi E}{\left(\hbar \omega_{g}\right)^{2}}\left(E-2 G_{n} \cos \left(q x_{0}\right)\right)\right\}\right]
\end{aligned}
$$

After first iteration, we have $\Gamma(E) / 2=\pi \Gamma_{0}^{2} E /\left(\hbar \omega_{g}\right)^{2}$, and then putting it back into Eq. (A5) and using $\epsilon=$ $E /\left(\hbar \omega_{g}\right)$, we get

$$
\begin{aligned}
\frac{\Gamma(\epsilon)}{2} & \simeq \frac{\pi \Gamma_{0}^{2} \epsilon}{\left(\hbar \omega_{g}\right)}\left[1+2 \exp \left\{-2\left(\frac{\pi \epsilon \Gamma_{0}}{\left(\hbar \omega_{g}\right)}\right)^{2}\right\}\right. \\
& \times \cos \left(\pi \epsilon^{2}\right) \\
& \left.\times\left\{1-\Omega_{m}\left(\epsilon \hbar \omega_{g}\right)^{3} \sin ^{2}\left(q l_{0} \epsilon-\frac{\pi}{4}\right)\right\}\right],
\end{aligned}
$$

where

$$
\Omega_{m}=\frac{\left(V_{m}^{g}\right)^{2}}{\pi^{2}}\left(\frac{a}{l_{0}}\right)^{3}\left(\frac{1}{\hbar \omega_{g}}\right)^{5} \sin ^{2}\left(\frac{q l_{0}}{2 \epsilon}\right)
$$

Using Eqs. (A3) and (A6), we get the density of states $D(\epsilon)$ as given in Eqs. (15) and (16).
1 A. H. Castro Neto, F. Guinea, N. M. R. Peres, K. S. Novoselov, and A. K. Geim, Rev. Mod. Phy. 81, 109 (2009).

2 K. S. Novoselov, A. K. Geim, S. V. Morozov, D. Jiang, Y. Zhang, S. V. Dubonos, I. V.Grigorieva, and A. A. Firsov, Science 306, 666 (2004).

3 Y. Zhang, Y. W. Tan, H. Stormer, and P. Kim, Nature (London) 438, 201 (2005).

4 C. Berger, Z. Song, X. Li, X. Wu, N. Brown, C. Naud, D. Mayou, T. Li, J. Hass, A. N. Marchenkov, E. H. Konrad, P. N. First, and W. A. de Heer, Science 312, 1191 (2006).

${ }^{5}$ K. S. Novoselov, A. k. Geim, S. V. Morozov, D. Jiang, M. I. Katsnelson, I. V. Grigorieva, S. V. Dubonos, and A. A.
Firsov, Nature 438, 197 (2005).

6 M. I. Katsnelson, K. S. Novoselov, and A. K. Geim, Nat. Phys. 2, 620 (2006).

7 K. S. Novoselov, E. McCann, S. V. Morozov, V. I. Falko, M. I. Katsnelson, U. Zeitler, D. Ziang, F. Schedin, and A. K. Geim, Nat. Phys. 2, 177 (2006).

8 Y. Zhang and T. Ando, Phys. Rev. B 65, 245420 (2002).

9 V. P. Gusynin and S. G. Sharapov, Phys. Rev. Lett. 95 146801 (2005).

10 D. Weiss, K. von Klitzing, K. Ploog, and G. Weinmann, Euro. Phys. Lett. 8, 179 (1989).

11 R. W. Winkler, J. P. Kotthaus, and K. Ploog, Phys. Rev. Lett. 62, 1177 (1989). 
12 F. M. Peters and P. Vasilopoulos, Phys. Rev. B 46, 4667, (1992).

13 R. R. Gerhardts, D. Weiss and K. von Klitzing, Phys. Rev. Lett. 62, 1173 (1989).

14 C. W. J. Beenakker, Phys. Rev. Lett. 62, 2020 (1989).

15 X. F. Wang, P. Vasilopoulos, and F. M. Peteers, Phys. Rev. B 71, 125301 (2005).

16 F. M. Peters and P. Vasilopoulos, Phys. Rev. B 47, 1466 (1993).

17 P. Vasilopoulos and F. M. Peters, Superlattices and Microstructures 7, 4 (1990).

18 P. Vasilopoulos and F. M. Peeters, Physica Scripta T 39, 177 (1991).

19 T. J. Li, Shi-Wei Gu, X. H. Wang and Jian Ping-Peng, J. Phys.: Condens. Matter 8, 313 (1996).

20 S. Izawa, S. Katsumoto, A. Endo, and Y. Iye, J. Phys. Soc. Jpn. 64, 706 (1995).

21 H. A. Carmona, A. K. Geim, A. Nogaret, P. C. Main, T. J. Foster, M. Heini, S. P. Beaumont, and M. G. Blamire, Phys. Rev. Lett. 74, 3009 (1995).

${ }^{22}$ P. D. Ye, D. Weiss, R. R. Gerhardts, M. Seeger, K. von Klitzing, K. Eberl, and H. Nickel, Phys. Rev. Lett. 74,
3013 (1995).

23 A. Matulis and F. M. Peters, Phy. Rev. B 75, 125429 (2007).

24 R. Nasir, K. Sabeeh, and M. Tahir, Phy. Rev. 81, 085402 (2010).

25 M. Tahir and K. Sabeeh, Phy. Rev. B 77, 195421 (2008).

26 S. M. Stewart and Chao Zhang, J. Phys.: Condens. Matter 10, 5545 (1998).

27 T. Jhong Li, Ke-lin Wang, and Jin-long Wang, J. Phys.: Condens. Matter 9, 9299 (1997).

28 R. Nasir, M. A. Khan, M. Tahir, and K. Sabeeh, J. Phys.: Condens. Matter 22, 025503 (2010).

29 M. Tahir and K. Sabeeh, Int. J. Mod. Phys. 23, 3445 (2009).

30 R. K. Pathria, Statistical Mechanics (ButterworthHeinemann, 2001).

31 C. Zhang and Rolf R. Gerhardts, Phys. Rev. B 41, 128550 (1990).

32 E. N. Economou, Green's functions in Quantum physics (Springer-Verlag, Berlin, 1990). 